\title{
Éditorial : Construire une bibliothèque des pratiques pédagogiques en anglais de spécialité
}

Editorial: Building a library of pedagogical practices in English for specific purposes

\section{Anthony Saber}

\section{OpenEdition}

\section{Journals}

Édition électronique

URL : http://journals.openedition.org/asp/5092

DOI : 10.4000/asp.5092

ISSN : 2108-6354

Éditeur

Groupe d'étude et de recherche en anglais de spécialité

Édition imprimée

Date de publication : 1 mars 2018

Pagination : 1-3

ISSN : 1246-8185

\section{Référence électronique}

Anthony Saber, «Éditorial : Construire une bibliothèque des pratiques pédagogiques en anglais de spécialité », ASp [En ligne], 73 | 2018, mis en ligne le 01 mars 2018, consulté le 01 novembre 2020

URL : http://journals.openedition.org/asp/5092 ; DOI : https://doi.org/10.4000/asp.5092

Ce document a été généré automatiquement le 1 novembre 2020

Tous droits réservés 


\title{
Éditorial : Construire une bibliothèque des pratiques pédagogiques en anglais de spécialité
}

\author{
Editorial : Building a library of pedagogical practices in English for specific
} purposes

Anthony Saber

1 «Trop de volumes et pas assez de livres » : cette formule paradoxale et malicieuse, attribuée à l'auteur de vaudevilles Adrien Decourcellle, pourrait illustrer le paysage actuel des pratiques pédagogiques en secteur LANSAD. Chaque jour, au sein de leur institution de rattachement, les enseignants d'anglais de spécialité imaginent des procédures d'enseignement originales, créent des exercices inventifs, et, attentifs aux besoins de leurs élèves, mettent en œuvre des approches spécifiques, écrivant ainsi au quotidien la réalité de notre discipline. Cependant, ces pratiques souvent innovantes ne sont que trop rarement archivées ou transmises à la communauté des enseignants LANSAD, ne bénéficiant que d'une diffusion limitée et locale, alors que leur indexation au sein d'une bibliothèque de référence serait incontestablement d'une grande utilité. C'est donc tout un continent d'actions et de dispositifs pédagogiques qui demeure, malheureusement, une vaste terra incognita.

2 À ce titre, l'équipe éditoriale est heureuse de vous annoncer le lancement d'une nouvelle rubrique dans notre revue, intitulée «pratiques pédagogiques en anglais de spécialité ", à compter du prochain numéro ${ }^{1}$. Issue d'une réflexion menée par Valérie Braud, Monique Mémet et Philippe Millot, elle aura vocation à répertorier et à archiver des pratiques pédagogiques remarquables, qui illustrent la créativité, les réflexions et le savoir-faire des praticiens du secteur LANSAD. La trame commune proposée aux auteurs pour rédiger ces rapports en provenance des différents «terrains» de notre discipline permettra aussi, nous l'espérons, de favoriser la comparaison des pratiques, et ainsi de contribuer à une meilleure codification de nos 
interventions pédagogiques en fonction du contexte institutionnel et des besoins des différentes populations en matière d'anglais de spécialité. Ces comptes rendus pédagogiques, d'une longueur préconisée de 4000 mots, comprendront ainsi des indications sur le dispositif de formation, les objectifs du cours, l'ingénierie des séances, l'évaluation de la progression, les procédures de notation ou de certification, ainsi qu'un bref bilan général de l'action de formation. Chaque contribution sera précédée d'un commentaire proposé par un enseignant-expert du secteur LANSAD, qui mettra en perspective l'objet traité par l'auteur. Nous invitons vivement tous nos collègues, notamment les agrégés et certifiés impliqués dans des formations en anglais de spécialité, à nous proposer des textes relatant leurs pratiques, quel que soit le contexte dans lequel ils travaillent (universités, grandes écoles, IUT, BTS, formation continue...).

3 Nous formons l'espoir que cette bibliothèque des pratiques pédagogiques en anglais de spécialité, certes potentiellement infinie sur un mode borgésien ${ }^{2}$, si l'on considère le très grand nombre de situations et de contextes, ainsi que la diversité des acteurs et des enjeux en secteur LANSAD, suscitera au fil du temps la construction collective de doctrines d'action et de procédures partagées. Ainsi, pour reprendre les derniers mots de Jorge Luis Borges dans sa nouvelle "La bibliothèque de Babel", "le désordre apparent, se répétant, constituerait un ordre, l'Ordre. Ma solitude se console à cet élégant espoir ».

4 Le présent numéro d'ASp comprend quatre articles de recherche. Adoptant un point de vue diachronique, Elizabeth Rowley-Jolivet s'intéresse à la multimodalité de communication au sein de la revue médicale The Lancet de 1823 à 2015. Si les articles publiés dans cette revue ont toujours comporté des éléments graphiques (par exemple des dessins illustrant des gestes chirurgicaux dans les articles publiés au XIX ${ }^{\mathrm{e}}$ siècle), c'est dans les années 1950, avec l'emprise croissante du modèle IMRAD, que les composants visuels (graphiques, tableaux, images, photographies...) se diversifient et se multiplient, souvent assortis de textes d'accompagnement de plus en plus détaillés, ce qui favorise une consultation non séquentielle des articles de recherche en médecine par les lecteurs experts. Cette évolution traduit le passage graduel d'une médecine d'inspiration principalement chirurgicale et narrative au XIX ${ }^{\text {e }}$ siècle, attachée à l'anatomie et à la description de cas individuels de patients, à une médecine de laboratoire de plus en plus axée sur les éléments de preuve statistiques.

5 Claire Kloppmann-Lambert réfléchit sur le rôle majeur de la métaphore chez les architectes s'exprimant dans The Architectural Review. Les réseaux métaphoriques mis en évidence traduisent le mode de pensée analogique de cette profession, qui conceptualise les bâtiments comme des êtres vivants, des plantes, des objets, des matériaux, voire, de manière plus surprenante encore, comme des textes, des textiles. Les métaphores sont dotées d'une dimension explicative qui traduit le projet architectural, et illustrent le double positionnement de l'architecte, qui est tantôt celui qui fait et conçoit (architectus ingenio), tantôt celui qui parle et explique (architectus verborus) ${ }^{3}$. Certaines métaphores sont même constitutives de théories architecturales actuelles, comme le «biomorphisme ». L'emploi très fréquent de ce trope par les acteurs du domaine, et la poésie qu'on note dans des expressions imagées telles que wet palimpsest ou geological nougat, traduit un tropisme artistique qui semble l'emporter sur une conception purement technicienne de l'architecture, inscrivant nettement celle-ci dans la constellation des arts plastiques. 
6 Adam Wilson tente de modéliser les interactions en anglais entre le personnel de l'office du tourisme de Marseille et les touristes visitant la cité phocéenne, inscrivant son étude au croisement de l'ethnographie, de la pragmatique, et de la caractérisation de l'anglais du tourisme. Le vecteur des échanges est bien entendu l'anglais lingua franca, qui permet aux interactants de se comprendre même si la forme du message est imparfaite. L'auteur met en relief plusieurs stratégies à l'œuvre dans les échanges : vérification de la compréhension par l'interlocuteur, confirmation du fait que l'information demandée a bien été fournie, signalement d'obstacles à la compréhension, «réparation» de quiproquos. Répétition et reformulation sont les principaux moyens langagiers de ces stratégies, qui relèvent d'une co-construction du sens, certes souvent laborieuse, par les interactants.

Laura-May Simard présente les résultats d'une étude de terrain portant sur un groupe de vingt-quatre étudiants en Master suivant un cours de rédaction scientifique anglaise. La moitié de cet effectif était invitée à utiliser de manière privilégiée un corpus et un concordancier comme principale ressource rédactionnelle, alors que l'autre demi-groupe devait avoir recours au dictionnaire en ligne Word Reference. L'analyse qualitative des productions écrites ainsi que des journaux de bord tenus par les étudiants démontre une bonne appropriation du couple corpus-concordancier par le premier groupe. Un gain d'efficacité rédactionnelle perceptible, mais modéré est noté pour les étudiants de niveau moyen ayant eu recours au corpus ; il est nettement plus prononcé pour les sujets bénéficiant déjà d'une bonne maîtrise linguistique. Une typologie des usages du corpus (recherche simple, recherche de collocation, vérification de formulations) par les étudiants est présentée dans cette étude, qui n'écarte cependant pas le recours au dictionnaire comme outil pédagogique en cours de rédaction scientifique.

Vous trouverez aussi dans ce numéro trois recensions par Adam Wilson, Shona Whyte et Anne-Marie Barrault-Méthy.

\section{NOTES}

1. Voir pages 125-127 de ce numéro et <http://journals.openedition.org/asp/5085>.

2. Comme l'on sait, Jorge Luis Borges formule en 1941, dans sa célèbre nouvelle « La Bibliothèque de Babel ", à la suite du philosophe allemand Kurd Lasswitz en 1904, l'hypothèse d'une bibliothèque qui contiendrait tous les ouvrages déjà écrits et tous ceux à venir.

3. On doit cette distinction à Caballero, Rosario. 2006. Re-viewing Space. Figurative Language in Architects' Assessment of Built Space. Berlin, New York : Mouton de Gruyter. 


\section{AUTEUR}

\section{ANTHONY SABER}

Rédacteur en chef. anthony.saber@ens-paris-saclay.fr 